\title{
fastQ_brew: module for analysis, preprocessing, and reformatting of FASTQ sequence data
}

Damien M. O'Halloran ${ }^{1,2^{*}}$ (D)

\begin{abstract}
Background: Next generation sequencing datasets are stored as FASTQ formatted files. In order to avoid downstream artefacts, it is critical to implement a robust preprocessing protocol of the FASTQ sequence in order to determine the integrity and quality of the data.

Results: Here I describe fastQ_brew which is a package that provides a suite of methods to evaluate sequence data in FASTQ format and efficiently implements a variety of manipulations to filter sequence data by size, quality and/or sequence. fastQ_brew allows for mismatch searches to adapter sequences, left and right end trimming, removal of duplicate reads, as well as reads containing non-designated bases. fastQ_brew also returns summary statistics on the unfiltered and filtered FASTQ data, and offers FASTQ to FASTA conversion as well as FASTQ reverse complement and DNA to RNA manipulations.
\end{abstract}

Conclusions: fastQ_brew is open source and freely available to all users at the following webpage: https://github. com/dohalloran/fastQ_brew.

Keywords: FASTQ, NGS, Sequencing

\section{Background}

FASTQ format has become the principal protocol for the exchange of DNA sequencing files [1]. The format is composed of both a nucleotide sequence as well as an ASCII character encoded quality score for each nucleotide. Each entry is four lines, with the first line starting with a '@' character followed by an identifier. The second line is the nucleotide sequence. The third line starts with a ' + ' character and optionally followed by the same sequence identifier that was used on the first line. The fourth line lists the quality scores for each nucleotide in the second line. In order to evaluate the quality of the FASTQ dataset and to avoid downstream artefacts, it is imperative for the user to employ robust quality control and preprocessing steps prior to downstream FASTQ applications.

\footnotetext{
*Correspondence: damienoh@gwu.edu

2 Department of Biological Sciences, The George Washington University, 636 Ross Hall, 2300 I St. N.W., Washington, DC 20052, USA

Full list of author information is available at the end of the article
}

Furthermore, FASTQ has now become widely used in additional downstream applications and pipelines, and so diverse preprocessing tools are necessary to handle various FASTQ file manipulations [2, 3]. Here, I describe fastQ_brew, which is a robust package that performs quality control, reformatting, filtering, and trimming of FASTQ formatted sequence datasets.

\section{Implementation}

fastQ_brew was developed using Perl and successfully tested on Microsoft Windows 7 Enterprise ver.6.1, Linux Ubuntu 64-bit ver.16.04 LTS, and Linux Mint 18.1 Serena. fastQ_brew does not rely on any dependencies that are not currently part of the Perl Core Modules (http:// perldoc.perl.org/index-modules-A.html), which makes fastQ_brew very straight forward to implement. fast $Q_{-}$ brew is composed of two separate packages: fastQ_brew. pm and fastQ_brew_Utilities.pm. fastQ_brew_Utilities. pm provides fastQ_brew.pm with access to various subroutines that are called to handle FASTQ manipulations 
and quality control. The fastQ_brew object is instantiated by calling the constructor subroutine called "new" which creates a 'blessed' object that begins gathering methods and properties by calling the load_fastQ_brew method. Once the object has been populated, the user can call run_fastQ_brew to begin processing the FASTQ data. Sample data are provided at the GitHub repo and directions for usage are described in the README.md file.

The command-line arguments supplied to the fast $Q_{-}$ brew object are as follows: (1) -lib, which can be either sanger or illumina; (2) -path, specifies the path to the input file (can use ".I" for current directory with UNIX or ".।" on Windows cmd); (3) $-i$, this is the name of the file containing the FASTQ reads; (4) -smry, return summary statistics table on the unfiltered data and filtered data; (5) $-q f$, this option will filter reads by Phred (also called Q score) quality score-any reads having an average Phred score below the threshold will be removed: e.g. $-q f=20$ will remove reads with Phred scores below 20; (6) $-l f$, this will filter reads below a specified length; (7) -trim_l, will trim the specified number of bases from the left end of each read; (8) -trim_ $r$, same as left-trim except that here the reads will be trimmed from the right side; (9) -adpt_l, will remove a specified adapter sequence from the left end of a read; (10) -adpt_r, same as -adpt_l except that here the reads will be trimmed from the right side; (11) -mis_l, allows for a specified number of mismatches between the user provided -adpt_l sequence and each read e.g. a mismatch $=1$, would match a hypothetical 3 base adapter, TAG, to the left end of a sequence that started with TAG or AAG or TAA or any of the nine possibilities; (12) -mis_r, same as -mis_l except that this relates to the $a d p t \_r$ sequence supplied by the user; (13) -dup, removes duplicate reads; (14) -no_n, removes reads that contain non-designated bases i.e. bases that are not A, G, C or T e.g. N; (15) -fasta, this option will convert the FASTQ file to FASTA format; (16) -rev_comp, will reverse complement reads in the supplied FASTQ file; (17) -rna, will convert each read to the corresponding RNA sequence in the supplied FASTQ file; (18) -clean, option to delete temporary files created during the run. If the summary option is selected, fastQ_brew will return a results table to STDOUT with summary statistics of the FASTQ data file prior to filtering and after filtering. The summary report will provide a table detailing max, min, and average GC\% values for all reads; max, min, and average read lengths, max, min, and average Phred scores, and max, min, and average error probabilities. The Phred score (denoted as $\mathrm{Q}$ ) represents the probability of an error for each base, and is logarithmically related to the basecalling error probability, $P$ such that:

$$
Q=-10 \log _{10} P
$$

or

$$
P=10 \frac{-Q}{10}
$$

In the case of arguments 15-17 above, a new file will be generated in each case, whereas for all other options the user-supplied arguments will be chained together to return a single filtered file.

\section{Results}

Testing of fastQ_brew was performed by plotting runtime against file size (Fig. 1a). FASTQ formatted sequence data from $110 \mathrm{MB}(462,664$ reads) to $4.5 \mathrm{~GB}(24,159,698$ reads) in size were used to benchmark the runtime of fastQ_brew. In each case, fastQ_brew efficiently returned summary statistics from each file in $36 \mathrm{~s}$ for $110 \mathrm{MB}$ FASTQ file to $25 \mathrm{~min}$ and $33 \mathrm{~s}$ for $4.5 \mathrm{~GB}$. The runtime will scale with the number of methods called within fastQ_brew.

To evaluate more specific methods within fastQ_brew, the relationship between nucleotide position within a given read and the corresponding Phred quality score was determined (Fig. 1b). This method tested the trimming and Phred calculation methods within fastQ_brew. The Phred quality score is used as a metric to determine the quality of a given nucleotide's identification within a read [4]. Phred quality scores are related (logarithmically) to the base-calling error probabilities [5] (see equation above). The average Phred quality scores for a randomly chosen FASTQ data file after left-side trimming (-trim_l) method invocations within fastQ_brew from position 1-20 were plotted (Fig. 1b). There was a negative correlation between increasing nucleotide position and Phred quality score $\left(R^{2}=-0.99969\right)$, that is, bases closer to the beginning of each read exhibit higher Phred quality scores, as compared with nucleotides closer to the middle of the read. This observation is in keeping with previous observations on Phred quality across reads [6-8] (http:// www.bioinformatics.babraham.ac.uk/projects/fastqc/). The data set used in this test was comprised of 462,664 reads with an average read length of 99 bases. The smallest read length was 25 bases and the largest was 100 bases.

To further examine the quality filtering method of fastQ_brew, FASTQ data were downloaded from the NCBI sequence read archive (SRA-https://www.ncbi. nlm.nih.gov/sra) using the sra-toolkit (https://github. com/ncbi/sra-tools). Distribution of read quality was plotted prior to filtering (blue bars) and after filtering (red bars) using fastQ_brew revealing a shift in Phred scores towards increased quality after filtering (Fig. 1c).

Finally, to compare fastQ_brew to other FASTQ filtering tools, I examined the execution time for some of the most commonly used filtering tools in trimming FASTQ data, and compared their execution speeds to that of 

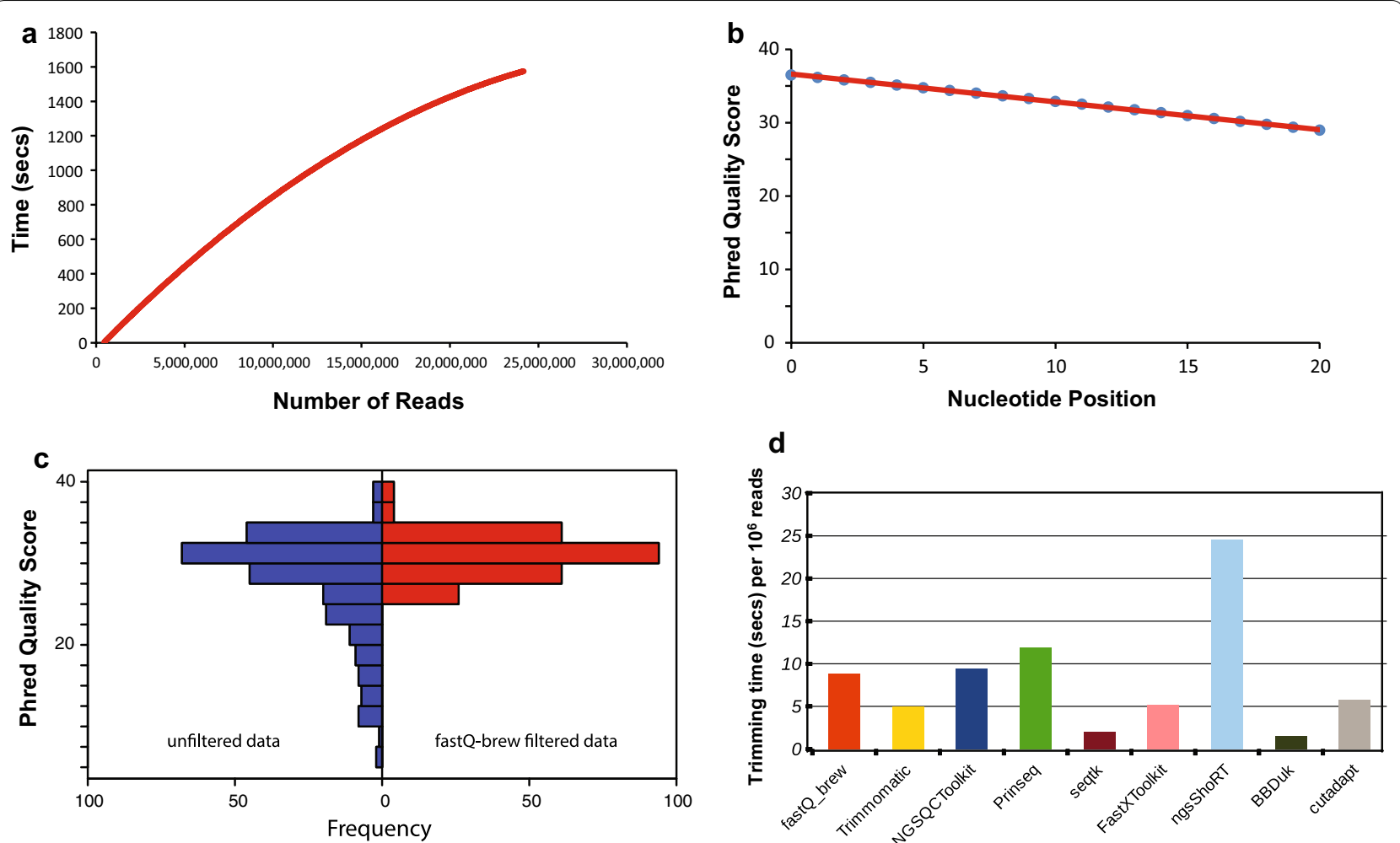

Fig. 1 a Performance testing of fastQ_brew. FASTQ formatted files containing different numbers of reads (110 MB [462,664 reads] to 4.5 GB $[24,159,698$ reads]) were provided as input to fastQ_brew and ran using default settings to return summary statistics for each dataset. b Relationship between nucleotide position and Phred quality score. fastQ_brew was used to determine the average Phred quality score from a FASTQ dataset comprising 462,664 reads after the length trimming methods was invoked to trim each read from position 1-20. A negative correlation between increasing nucleotide position and quality was observed. c The quality filter method within fastQ_brew was tested by plotting the Phred scores before (blue bars) and after (red bars) quality filtering. After filtering, there was a shift in the distribution of reads towards higher quality Phred values. d Execution speed for commonly used FASTQ filtering tools were compared with fastQ_brew. For all analyses, the same file and trimming task was applied. The following software were compared and presented: fasta_brew ver 1.0.2, trimmomatic ver 0.36, NGSQCToolkit ver 2.3.3, Prinseq ver 0.20.4, seatk, Fastxtoolkit ver 0.0.13, ngsShoRT ver 2.2, BBDuk ver 37.22, and Cutadapt ver 1.9.1

fastQ_brew. For all analyses, the same FASTQ file was used, and in each case methods were invoked to trim 8 bases from the left and right sides of every read in the file. The following software were used: fastq_brew ver 1.0.2; Trimmomatic ver 0.36 [9]; NGSQCToolkit ver 2.3 .3 [6]; Prinseq ver 0.20.4 [10]; seqtk (https://github.com/ lh3/seqtk); Fastxtoolkit ver 0.0.13 (http://hannonlab.cshl. edu/fastx_toolkit/index.html); BBDuk ver 37.22 (http:// jgi.doe.gov/data-and-tools/bbtools/bb-tools-user-guide/ bbmap-guide/); ngsShoRT ver 2.2 [11]; and Cutadapt ver 1.9.1 (http://journal.embnet.org/index.php/embnetjournal/article/view/200). For some other software tools, this exact invocation was not possible due to limitations on the trimming method. The data from this analysis is presented in Fig. 1d. fastQ_brew compares well with other commonly employed filtering tools. The fastest tool was $B B D u k$ which finished trimming all reads in only $1.532 \mathrm{~s}$, and this was followed very closely by seqtk which completed the task in $1.99 \mathrm{~s}$. By examining across these tools we can obtain some insight into how the execution speeds for fastQ_brew compares with commonly used trimming software. However, it is important to point out that each tool offers many specific adaptations and features that are not reflected in a basic trimming task, and while speed is important when dealing with very large data-sets, other features that include accessibility, documentation, ease of use, as well as applicability of options are equally important.

In summary, I here describe fastQ_brew, a very lightweight Perl package for robust analysis, preprocessing, and manipulation of FASTQ sequence data files. The main advantage of fastQ_brew is its ease of use, as the software does not rely on any modules that are not currently contained within the Perl Core. fastQ_brew is freely available on GitHub at: https://github.com/ dohalloran/fastQ_brew. 


\section{Author details}

${ }^{1}$ Institute for Neuroscience, The George Washington University, 636 Ross Hall, 2300 I St. N.W., Washington, DC 20052, USA. ${ }^{2}$ Department of Biological Sciences, The George Washington University, 636 Ross Hall, 2300 I St. N.W., Washington, DC 20052, USA.

\section{Acknowledgements}

I thank members of the O'Halloran lab for critical reading of the manuscript.

\section{Competing interests}

The author declares no competing interests.

\section{Availability of data and materials}

Project name: fastQ_brew.

Project home page: https://github.com/dohalloran/fastQ_brew.

Operating system(s): Platform independent.

Programming language: Perl.

Other requirements: none.

License: GNU.

Any restrictions to use by non-academics: no restrictions or login requirements.

\section{Funding}

The George Washington University (GWU) Columbian College of Arts and Sciences, GWU Office of the Vice-President for Research, and the GWU Department of Biological Sciences.

\section{Publisher's Note}

Springer Nature remains neutral with regard to jurisdictional claims in published maps and institutional affiliations.

Received: 19 January 2017 Accepted: 8 July 2017

Published online: 12 July 2017

\section{References}

1. Cock PJ, Fields CJ, Goto N, Heuer ML, Rice PM. The Sanger FASTQ file format for sequences with quality scores, and the Solexa/Illumina FASTQ variants. Nucleic Acids Res. 2010;38(6):1767-71.

2. Kim M, Zhang X, Ligo JG, Farnoud F, Veeravalli W, Milenkovic O. MetaCRAM: an integrated pipeline for metagenomic taxonomy identification and compression. BMC Bioinform. 2016;17:94. doi:10.1186/ s12859-016-0932-x.

3. Ferraro Petrillo U, Roscigno G, Cattaneo G, Giancarlo R. FASTdoop: a versatile and efficient library for the input of FASTA and FASTQ files for MapReduce Hadoop bioinformatics applications. Bioinformatics. 2017;33:1575-7.

4. Ewing B, Hillier L, Wendl MC, Green P. Base-calling of automated sequencer traces using phred. I. Accuracy assessment. Genome Res. 1998;8(3):175-85.

5. Ewing B, Green P. Base-calling of automated sequencer traces using phred. II. Error probabilities. Genome Res. 1998;8(3):186-94.

6. Patel RK, Jain M. NGS QC toolkit: a toolkit for quality control of next generation sequencing data. PLoS ONE. 2012;7(2):e30619.

7. Schmieder R, Lim YW, Rohwer F, Edwards R. TagCleaner: identification and removal of tag sequences from genomic and metagenomic datasets. BMC Bioinform. 2010;11:341. doi:10.1186/1471-2105-11-341.

8. Cox MP, Peterson DA, Biggs PJ. SolexaQA: at-a-glance quality assessment of Illumina second-generation sequencing data. BMC Bioinform. 2010;11:485. doi:10.1186/1471-2105-11-485.

9. Bolger AM, Lohse M, Usadel B. Trimmomatic: a flexible trimmer for illumina sequence data. Bioinformatics. 2014;30(15):2114-20.

10. Schmieder R, Edwards R. Quality control and preprocessing of metagenomic datasets. Bioinformatics. 2011;27(6):863-4.

11. Chen C, Khaleel SS, Huang H, Wu CH. Software for pre-processing illumina next-generation sequencing short read sequences. Source Code Biol Med. 2014;9:8. doi:10.1186/1751-0473-9-8.

\section{Submit your next manuscript to BioMed Central and we will help you at every step:}

- We accept pre-submission inquiries

- Our selector tool helps you to find the most relevant journal

- We provide round the clock customer support

- Convenient online submission

- Thorough peer review

- Inclusion in PubMed and all major indexing services

- Maximum visibility for your research

Submit your manuscript at www.biomedcentral.com/submit 